\title{
SHARP INEQUALITIES FOR ANTI-INVARIANT RIEMANNIAN SUBMERSIONS FROM SASAKIAN SPACE FORMS
}

\author{
HÜLYA AYTIMUR AND CIHAN ÖZGÜR
}

\begin{abstract}
We obtain sharp inequalities involving the Ricci curvature and the scalar curvature for anti-invariant Riemannian submersions from Sasakian space forms onto Riemannian manifolds.
\end{abstract}

\section{Introduction}

To find relationship between the extrinsic and intrinsic invariants of a submanifold have been very popular problems in the recent twenty five years. The first study in this direction was started by B.-Y. Chen in 1993. He established some inequalities between the main extrinsic (the squared mean curvature) and main intrinsic invariants (the scalar curvature and the Ricci curvature) of a submanifold in a real space form [11. In 1999, Chen also established a relation between the Ricci curvature and the squared mean curvature for a submanifold [12]. After that, many papers have been published by various authors in different ambient spaces. In 2011, Chen published a book which consists of the all studies doing in these directions [13]. The topic is still very popular and there are many new papers related to the inequalities which are introduced by Chen. For example see [2], [10], [12], [15], [16], [17], [18, [19] and [21].

Let $(M, g)$ and $\left(B, g^{\prime}\right)$ be $m$ and $b$-dimensional Riemannian manifolds, respectively. A Riemannian submersion $\pi: M \rightarrow B$ is a mapping of $M$ onto $B$ such that $\pi$ has a maximal rank and the differential $\pi_{*}$ preserves the lengths of the horizontal vectors [8]. In [4] and [5], Chen proved a simple optimal relationship between Riemannian submersions and minimal immersions [4]. In [1, Alegre, Chen and Munteanu established a sharp relationship between the $\delta$-invariants and Riemannian submersions with totally geodesic fibers. In [7], Gülbahar, Meriç and Kılıç obtained sharp inequalities involving the Ricci curvature for Riemannian

2010 Mathematics Subject Classification. 53C40, 53B05, 53B15, 53C05, 53A40.

Key words and phrases. Sasakian space form, Riemannian submersion, anti-invariant Riemannian submersion, Chen-Ricci inequality. 
submersions. In [20], Şahin introduced anti-invariant Riemannian submersions from almost Hermitian manifolds onto Riemannian manifolds.

Motivated by the above studies, in the present study, we consider anti-invariant Riemannian submersions from Sasakian manifolds onto Riemannian manifolds. We obtain sharp inequalities involving the Ricci curvature and the scalar curvature.

The paper is organized as follows. In Section 2, we give brief introduction about Sasakian manifolds and submersions. We give some lemmas which will be used in Section 3 and Section 4. In Section 3, we obtain some inequalities involving the Ricci curvature and the scalar curvature on the vertical and horizontal distributions for anti-invariant Riemannian submersions from Sasakian space forms. The equality cases are also discussed. In Section 4, we prove Chen-Ricci inequalities on the vertical and horizontal distributions for anti-invariant Riemannian submersions from Sasakian space forms. We find relationships between the intrinsic and extrinsic invariants using fundamental tensors. The equality cases are also considered.

\section{Preliminaries}

Let $\pi: M \rightarrow B$ be a Riemannian submersion. We put $\operatorname{dim} M=2 m+1$ and $\operatorname{dim} B=b$. For $x \in B$, Riemannian submanifold $\pi^{-1}(x)$ with the induced metric $\bar{g}$ is called a fiber and denoted by $\bar{M}$. We notice that the dimension of each fiber is always $(2 m+1-b)=r$ and dimension of the horizontal distribution is $n=(2 m+1-r)$. In the tangent bundle $T M$ of $M$, the vertical and horizontal distributions are denoted by $\mathcal{V}(M)$ and $\mathcal{H}(M)$, respectively. We call a vector field $X$ on $M$ projectable if there exists a vector field $X_{*}$ on $B$ such that $\pi_{*}\left(X_{p}\right)=$ $X_{* \pi(p)}$ for each $p \in M$. In this case, we call that $X$ and $X_{*}$ are $\pi$-related. A vector field $X$ on $M$ is called basic if it is projectable and horizontal ([8] and [9]).

The tensor fields $T$ and $A$ of type $(1,2)$ are defined by

$$
\begin{aligned}
& T_{E} F=h \nabla_{v E} v F+v \nabla_{v E} h F, \\
& A_{E} F=h \nabla_{h E} v F+v \nabla_{h E} h F .
\end{aligned}
$$

Denote by $R, R^{\prime}, \widehat{R}$ and $R^{*}$ the Riemannian curvature tensor of Riemannian manifolds $M, B$, the vertical distribution $\mathcal{V}$ and the horizontal distribution $\mathcal{H}$, 
respectively. Then the Gauss-Codazzi type equations are given by

$$
\begin{gathered}
R(U, V, F, W)=\widehat{R}(U, V, F, W)+g\left(T_{U} W, T_{V} F\right)-g\left(T_{V} W, T_{U} F\right) \\
R(X, Y, Z, H)=R^{*}(X, Y, Z, H)-2 g\left(A_{X} Y, A_{Z} H\right) \\
+g\left(A_{Y} Z, A_{X} H\right)-\left(A_{X} Z, A_{Y} H\right) \\
R(X, V, Y, W)=g\left(\left(\nabla_{X} T\right)(V, W), Y\right)+g\left(\left(\nabla_{V} A\right)(X, Y), W\right) \\
-g\left(T_{V} X, T_{W} Y\right)+g\left(A_{Y} W, A_{X} V\right),
\end{gathered}
$$

where

$$
\pi_{*}\left(R^{*}(X, Y) Z\right)=R^{\prime}\left(\pi_{*} X, \pi_{*} Y\right) \pi_{*} Z
$$

for any $X, Y, Z, H \in \chi^{h}(M)$ and $U, V, F, W \in \chi^{v}(M)$ [8].

Moreover, the mean curvature vector field $H$ of any fibre of Riemannian submersion $\pi$ is given by

$$
H=r N, \quad N=\sum_{j=1}^{r} T_{U_{j}} U_{j}
$$

where $\left\{U_{1}, \ldots, U_{r}\right\}$ is an orthonormal basis of the vertical distribution $\mathcal{V}$. Furthermore, $\pi$ has totally geodesic fibers if $T$ vanishes on $\chi^{h}(M)$ and $\chi^{v}(M)$.

Now we give the following lemmas:

Lemma 2.1. [6] Let $(M, g)$ and $\left(B, g^{\prime}\right)$ be Riemannian manifolds admitting a Riemannian submersion $\pi: M \rightarrow B$. For $E, F, G \in \chi(M)$, we have

$$
\begin{aligned}
& g\left(T_{E} F, G\right)=-g\left(F, T_{E} G\right), \\
& g\left(A_{E} F, G\right)=-g\left(F, A_{E} G\right) .
\end{aligned}
$$

That is, $A_{E}$ and $T_{E}$ are anti-symmetric with respect to $g$.

Lemma 2.2. [6] Let $(M, g)$ and $\left(B, g^{\prime}\right)$ be Riemannian manifolds admitting a Riemannian submersion $\pi: M \rightarrow B$.

(i) For $U, V \in \chi^{v}(M)$,

$$
T_{U} V=T_{V} U
$$

(ii) For $X, Y \in \chi^{h}(M)$,

$$
A_{X} Y=-A_{Y} X
$$


Let $M$ be a $(2 m+1)$-dimensional manifold and $\phi, \xi, \eta$ a tensor field of type $(1,1)$, a vector field, a 1 -form on $M$, respectively. If $\phi, \xi$ and $\eta$ satisfy the following conditions

$$
\eta(\xi)=1, \quad \phi^{2} X=-X+\eta(X) \xi
$$

for $X \in T M$, then $M$ is said to have an almost contact structure $(\phi, \xi, \eta)$ and $(M, \phi, \xi, \eta)$ is called an almost contact manifold. If

$$
\nabla_{X} \xi=-\phi X, \quad\left(\nabla_{X} \phi\right) Y=g(X, Y) \xi-\eta(Y) X,
$$

then $(M, \nabla, g, \phi, \xi, \eta)$ is called a Sasakian manifold [3], where $\nabla$ denotes the Levi-Civita connection of $g . \phi$ is anti-symmetric with respect to $g$, that is, for $X, Y \in T M$

$$
g(\phi X, Y)+g(X, \phi Y)=0 .
$$

A plane section $\pi$ in $T M$ is called a $\phi$-section if it is spanned by $X$ and $\phi X$, where $X$ is a unit tangent vector field orthogonal to $\xi$. The sectional curvature of a $\phi$-section is called a $\phi$-sectional curvature. A Sasakian manifold with constant $\phi$-sectional curvature $c$ is said to be a Sasakian space form [3] and is denoted by $M(c)$. The curvature tensor $R$ of $M(c)$ is expressed by

$$
\begin{gathered}
R(X, Y) Z=\frac{c+3}{4}[g(Y, Z) X-g(X, Z) Y]+\frac{c-1}{4}[\eta(X) \eta(Z) Y \\
-\eta(Y) \eta(Z) X+g(X, Z) \eta(Y) \xi-g(Y, Z) \eta(X) \xi+g(\phi Y, Z) \phi X \\
-g(\phi X, Z) \phi Y-2 g(\phi X, Y) \phi Z] .
\end{gathered}
$$

Definition 2.1. [14] Let $(M, \nabla, g, \phi, \xi, \eta)$ be a Sasakian manifold and $\left(B, g^{\prime}\right)$ a Riemannian manifold. A Riemannian submersion $\pi: M \rightarrow B$ is called antiinvariant if $\mathcal{V}(M)$ is anti-invariant with respect to $\phi$, i.e. $\phi(\mathcal{V}(M)) \subseteq \mathcal{H}(M)$.

Let $\pi:(M, \nabla, g, \phi, \xi, \eta) \rightarrow\left(B, g^{\prime}\right)$ be an anti-invariant Riemannian submersion from a Sasakian manifold $(M, \nabla, g, \phi, \xi, \eta)$ to a Riemannian manifold $\left(B, g^{\prime}\right)$. From Definition 2.1, we have $\phi(\mathcal{V}(M)) \cap \mathcal{H}(M) \neq\{0\}$. We denote the complementary orthogonal distribution to $\phi(\mathcal{V}(M))$ in $\mathcal{H}(M)$ by $\mu$. Then we have

$$
\mathcal{H}(M)=\phi(\mathcal{V}(M)) \oplus \mu .
$$

Suppose that $\xi$ is vertical. It is easy to see that $\mu$ is an invariant distribution of $\mathcal{H}(M)$ under the endomorphism $\phi$. Thus for $X \in \chi^{h}(M)$, we write

$$
\phi X=B X+C X
$$


where $B X \in \chi^{v}(M)$ and $C X \in \chi(\mu)[14]$.

Suppose that $\xi$ is horizontal. It is easy to see that $\mu=\phi \mu \oplus\{\xi\}$. Thus for $X \in \chi^{h}(M)$, we write

$$
\phi X=B X+C X
$$

where $B X \in \chi^{v}(M)$ and $C X \in \chi(\mu)[14]$.

Lemma 2.3. [14] Let $\pi: M \rightarrow B$ be an anti-invariant Riemannian submersion from a Sasakian manifold $(M, \nabla, g, \phi, \xi, \eta)$ to a Riemannian manifold $\left(B, g^{\prime}\right)$.

(i) If $\xi$ is vertical, then $C^{2} X=-X-\phi B X$

(ii) If $\xi$ is horizontal, then $C^{2} X=-X+\eta(X) \xi-\phi B X$.

Example 2.1. 3] Let us take $M=\mathbb{R}^{2 m+1}$ with the standard coordinate functions $\left(x_{1}, \ldots, x_{m}, y_{1}, \ldots, y_{m}, z\right)$, the contact structure $\eta=\frac{1}{2}\left(d z-\sum_{i=1}^{m} y_{i} d x_{i}\right)$, the characteristic vector field $\xi=2 \frac{\partial}{\partial z}$ and the tensor field $\varphi$ given by

$$
\varphi=\left[\begin{array}{ccc}
0 & \delta_{i j} & 0 \\
-\delta_{i j} & 0 & 0 \\
0 & y_{j} & 0
\end{array}\right]
$$

The Riemannian metric is $g=\eta \otimes \eta+\frac{1}{4} \sum_{i=1}^{m}\left(\left(d x_{i}\right)^{2}+\left(d y_{i}\right)^{2}\right)$. Then $\left(M^{2 m+1}, \varphi, \xi, \eta, g\right)$ is a Sasakian space form with constant $\varphi$-sectional curvature $c=-3$ and it is denoted by $\mathbb{R}^{2 m+1}(-3)$. The vector fields

$$
E_{i}=2 \frac{\partial}{\partial y_{i}}, E_{i+m}=\varphi X_{i}=2\left(\frac{\partial}{\partial x_{i}}+y_{i} \frac{\partial}{\partial z}\right), 1 \leq i \leq m, \xi=2 \frac{\partial}{\partial z},
$$

form a g-orthonormal basis for the contact metric structure.

Example 2.2. [14] We consider $M=\mathbb{R}^{5}(-3)$ with the structure given in Example 2.1. The Riemannian metric $g_{\mathbb{R}^{2}}$ is given by

$$
g_{\mathbb{R}^{2}}=\frac{1}{8}\left[\begin{array}{ll}
1 & 0 \\
0 & 1
\end{array}\right]
$$

on $\mathbb{R}^{2}$. Let $\pi: \mathbb{R}^{5}(-3) \rightarrow \mathbb{R}^{2}$ be a map defined by

$$
\pi\left(x_{1}, x_{2}, y_{1}, y_{2}, z\right)=\left(x_{1}+y_{1}, x_{2}+y_{2}\right) .
$$

Then

$$
\mathcal{V}(M)=\operatorname{sp}\left\{V_{1}=E_{1}-E_{3}, V_{2}=E_{2}-E_{4}, V_{3}=E_{5}=\xi\right\}
$$


and

$$
\mathcal{H}(M)=\operatorname{sp}\left\{H_{1}=E_{1}+E_{3}, H_{2}=E_{2}+E_{4}\right\} .
$$

So $\pi$ is a Riemannian submersion. Moreover, $\phi V_{1}=H_{1}, \phi V_{2}=H_{2}, \phi V_{3}=0$ imply that $\phi(\mathcal{V}(M))=\mathcal{H}(M)$. Hence $\pi$ is an anti-invariant Riemannian submersion such that $\xi$ is vertical.

Example 2.3. [14] We consider $M=\mathbb{R}^{5}(-3)$ with the structure given in Example 2.1. Let $N=\mathbb{R}^{3}-\left\{\left(y_{1}, y_{2}, z\right) \in \mathbb{R}^{3} \mid y_{1}^{2}+y_{2}^{2} \leq 2\right\}$. The Riemannian metric tensor $g_{N}$ is given by

$$
g_{N}=\frac{1}{4}\left[\begin{array}{ccc}
\frac{1}{2} & \frac{y_{1} y_{2}}{2} & -\frac{y_{1}}{2} \\
\frac{y_{1} y_{2}}{2} & \frac{1}{2} & -\frac{y_{2}}{2} \\
-\frac{y_{1}}{2} & -\frac{y_{2}}{2} & 1
\end{array}\right]
$$

on $N$. Let $\pi: \mathbb{R}^{5}(-3) \rightarrow N$ be a map defined by

$$
\pi\left(x_{1}, x_{2}, y_{1}, y_{2}, z\right)=\left(x_{1}+y_{1}, x_{2}+y_{2}, \frac{y_{1}^{2}}{2}+\frac{y_{2}^{2}}{2}+z\right) \text {. }
$$

Then

$$
\mathcal{V}(M)=\operatorname{sp}\left\{V_{1}=E_{1}-E_{3}, V_{2}=E_{2}-E_{4}\right\}
$$

and

$$
\mathcal{H}(M)=\operatorname{sp}\left\{H_{1}=E_{1}+E_{3}, H_{2}=E_{2}+E_{4}, H_{3}=E_{5}=\xi\right\} .
$$

So $\pi$ is a Riemannian submersion. Moreover, $\phi V_{1}=H_{1}, \phi V_{2}=H_{2}$ imply that $\phi(\mathcal{V}(M)) \subset \mathcal{H}(M)=\phi(\mathcal{V}(M)) \oplus\{\xi\}$. Hence $\pi$ is an anti-invariant Riemannian submersion such that $\xi$ is horizontal.

\section{Inequalities for anti-invariant Riemannian submersions}

In the present section, we aim to obtain some inequalities involving the Ricci curvature and the scalar curvature on the vertical and horizontal distributions for anti-invariant Riemannian submersions from Sasakian space forms. We shall also consider the equality cases of these inequalities.

Let $(M(c), g),\left(B, g^{\prime}\right)$ be a Sasakian space form and a Riemannian manifold, respectively and $\pi: M(c) \rightarrow B$ an anti-invariant Riemannian submersion. Furthermore, let $\left\{U_{1}, \ldots, U_{r}, X_{1}, \ldots, X_{n}\right\}$ be an orthonormal basis of $T M(c)$ such that $\mathcal{V}=\operatorname{span}\left\{U_{1}, \ldots, U_{r}\right\}, \mathcal{H}=\operatorname{span}\left\{X_{1}, \ldots, X_{n}\right\}$. Then using (2.4) and (2.1), we have 


$$
\begin{gathered}
\widehat{R}(U, V, F, W)=\frac{c+3}{4}\{g(V, F) g(U, W)-g(U, F) g(V, W)\} \\
+\frac{c-1}{4}\{\eta(U) \eta(F) g(V, W)-\eta(V) \eta(F) g(U, W) \\
+\eta(V) \eta(W) g(U, F)-\eta(U) \eta(W) g(V, F)+g(\phi V, F) g(\phi U, W) \\
-g(\phi V, W) g(\phi U, F)-2 g(W, \phi F) g(\phi U, V)\} \\
-g\left(T_{U} W, T_{V} F\right)+g\left(T_{V} W, T_{U} F\right) .
\end{gathered}
$$

Similarly, from (2.4) and (2.2), we get

$$
\begin{gathered}
R^{*}(X, Y, Z, H)=\frac{c+3}{4}\{g(Y, Z) g(X, H)-g(X, Z) g(Y, H)\} \\
+\frac{c-1}{4}\{\eta(X) \eta(Z) g(Y, H)-\eta(Y) \eta(Z) g(X, H) \\
+\eta(Y) \eta(H) g(X, Z)-\eta(X) \eta(H) g(Y, Z)+g(\phi Y, Z) g(\phi X, H) \\
-g(\phi Y, H) g(\phi X, Z)-2 g(H, \phi Z) g(\phi X, Y)\} \\
+2 g\left(A_{X} Y, A_{Z} H\right)-g\left(A_{Y} Z, A_{X} H\right)+\left(A_{X} Z, A_{Y} H\right) .
\end{gathered}
$$

Case I: Assume that $\xi$ is vertical.

For the vertical distribution, in view of (3.1), since $\pi$ is anti-invariant and $\xi$ is vertical, we find

$$
\begin{gathered}
\widehat{\operatorname{Ric}}(U)=\frac{c+3}{4}(r-1) g(U, U)+\frac{c-1}{4}\left\{(2-r) \eta(U)^{2}-g(U, U)\right\} \\
-r g\left(T_{U} U, H\right)+\sum_{j=1}^{r} g\left(T_{U_{j}} U, T_{U} U_{j}\right) .
\end{gathered}
$$

Hence we obtain the following theorem:

Theorem 3.1. Let $\pi: M(c) \rightarrow B$ be an anti-invariant Riemannian submersion from a Sasakian space form $(M(c), g)$ onto a Riemannian manifold $\left(B, g^{\prime}\right)$ such that $\xi$ is vertical. Then

$$
\widehat{\operatorname{Ric}}(U) \geq \frac{c+3}{4}(r-1)-\frac{c-1}{4}\left\{(r-2) \eta(U)^{2}+1\right\}-r g\left(T_{U} U, H\right) .
$$

The equality case of the inequality holds for a unit vertical vector field $U \in$ $\chi^{\mathcal{V}}(M(c))$ if and only if each fiber is totally geodesic. 
Similarly in view of (3.1), using the symmetry of $T$, we have

$$
2 \widehat{\tau}=\frac{c+3}{4} r(r-1)+\frac{c-1}{4}(2-2 r)-r^{2}\|H\|^{2}+\sum_{i, j=1}^{r} g\left(T_{U_{i}} U_{j}, T_{U_{i}} U_{j}\right),
$$

where $\widehat{\tau}=\sum_{1 \leq i<j \leq r} \widehat{R}\left(U_{i}, U_{j}, U_{j}, U_{i}\right)$. Then we can write

$$
2 \widehat{\tau} \geq \frac{c+3}{4} r(r-1)-\frac{c-1}{2}(r-1)-r^{2}\|H\|^{2} .
$$

The equality case of the inequality holds if and only if $T=0$, which means that each fiber is totally geodesic. Thus we can state the following theorem:

Theorem 3.2. Let $\pi: M(c) \rightarrow B$ be an anti-invariant Riemannian submersion from a Sasakian space form $(M(c), g)$ onto a Riemannian manifold $\left(B, g^{\prime}\right)$ such that $\xi$ is vertical. Then

$$
2 \widehat{\tau} \geq \frac{c+3}{4} r(r-1)-\frac{c-1}{2}(r-1)-r^{2}\|H\|^{2} .
$$

The equality case of the inequality holds if and only if each fiber is totally geodesic.

For the horizontal distribution, in view of (3.2), since $\pi$ is anti-invariant and $\xi$ is vertical, using the anti-symmetry of $A$, we find

$$
\begin{gathered}
2 \tau^{*}=\frac{c+3}{4} n(n-1) \\
+\sum_{i, j=1}^{n}\left[\frac{3(c-1)}{4} g\left(C X_{i}, X_{j}\right) g\left(C X_{i}, X_{j}\right)-3 g\left(A_{X_{i}} X_{j}, A_{X_{i}} X_{j}\right)\right] .
\end{gathered}
$$

By the use of Lemma 2.3, we obtain

$$
2 \tau^{*}=\frac{c+3}{4} n(n-1)+\frac{3}{4}(c-1)(n+\operatorname{tr}(\phi B))-\sum_{i, j=1}^{n} 3 g\left(A_{X_{i}} X_{j}, A_{X_{i}} X_{j}\right) .
$$

Then we can write

$$
2 \tau^{*} \leq \frac{c+3}{4} n(n-1)+\frac{3}{4}(c-1)(n+\operatorname{tr}(\phi B)),
$$

where $\tau^{*}=\sum_{1 \leq i<j \leq n} R^{*}\left(X_{i}, X_{j}, X_{j}, X_{i}\right)$. The equality case of (3.4) holds if and only if $A=0$, which means that the horizontal distribution is integrable. So we can state the following theorem: 
Theorem 3.3. Let $\pi: M(c) \rightarrow B$ be an anti-invariant Riemannian submersion from a Sasakian space form $(M(c), g)$ onto a Riemannian manifold $\left(B, g^{\prime}\right)$ such that $\xi$ is vertical. Then

$$
2 \tau^{*} \leq \frac{c+3}{4} n(n-1)+\frac{3}{4}(c-1)(n+\operatorname{tr}(\phi B)) .
$$

The equality case of (3.4) holds if and only if $\mathcal{H}(M)$ is integrable.

Case II: Assume that $\xi$ is horizontal.

From (3.1), since $\pi$ is anti-invariant submersion, after some computations, we have

$$
2 \widehat{\tau}=\frac{c+3}{4} r(r-1)-r^{2}\|H\|^{2}+\sum_{i, j=1}^{r} g\left(T_{U_{i}} U_{j}, T_{U_{i}} U_{j}\right) .
$$

Hence we can state the following theorem:

Theorem 3.4. Let $\pi: M(c) \rightarrow B$ be an anti-invariant Riemannian submersion from a Sasakian space form $(M(c), g)$ onto a Riemannian manifold $\left(B, g^{\prime}\right)$ such that $\xi$ is horizontal. Then

$$
2 \widehat{\tau} \geq \frac{c+3}{4} r(r-1)-r^{2}\|H\|^{2} .
$$

The equality case of the inequality holds if and only if each fiber is totally geodesic.

For the horizontal distribution, from (3.2), since $\xi$ is horizontal and $A$ is antisymmetric, after some computations, we have

$$
\begin{aligned}
2 \tau^{*}=\frac{c+3}{4} n(n-1)+\sum_{i, j=1}^{n} & {\left[\frac{c-1}{4}\left\{2-2 n+3 g\left(C X_{i}, X_{j}\right) g\left(C X_{i}, X_{j}\right)\right\}\right.} \\
& \left.-3 g\left(A_{X_{i}} X_{j}, A_{X_{i}} X_{j}\right)\right] .
\end{aligned}
$$

Then using Lemma 2.3, we obtain

$$
\begin{gathered}
2 \tau^{*}=\frac{c+3}{4} n(n-1)+\frac{c-1}{4}(3 \operatorname{tr} \phi B+n-1) \\
-\sum_{i, j=1}^{n} 3 g\left(A_{X_{i}} X_{j}, A_{X_{i}} X_{j}\right),
\end{gathered}
$$

where $\tau^{*}=\sum_{1 \leq i<j \leq n} R^{*}\left(X_{i}, X_{j}, X_{j}, X_{i}\right)$.

So we can state the following theorem: 
Theorem 3.5. Let $\pi: M(c) \rightarrow B$ be an anti-invariant Riemannian submersion from a Sasakian space form $(M(c), g)$ onto a Riemannian manifold $\left(B, g^{\prime}\right)$ such that $\xi$ is horizontal. Then

$$
2 \tau^{*} \leq \frac{c+3}{4} n(n-1)+\frac{(c-1)}{4}(3 \operatorname{tr}(\phi B)+n-1) .
$$

The equality case of the inequality holds if and only if $\mathcal{H}(M)$ is integrable.

\section{Chen-Ricci inequalities for anti-invariant Riemannian submersions}

In the present section, we aim to obtain Chen-Ricci inequality on the vertical and horizontal distributions for anti-invariant Riemannian submersions from a Sasakian space forms onto a Riemannian manifold. The equality cases will be also considered.

Let $(M(c), g)$ be a Sasakian space form and $\left(B, g^{\prime}\right)$ a Riemannian manifold. Assume that $\pi: M(c) \rightarrow B$ is an anti-invariant Riemannian submersion and $\left\{U_{1}, \ldots, U_{r}, X_{1}, \ldots, X_{n}\right\}$ is an orthonormal basis of $T M(c)$ such that $\mathcal{V}=\operatorname{span}\left\{U_{1}, \ldots\right.$, $\left.U_{r}\right\}, \mathcal{H}=\operatorname{span}\left\{X_{1}, \ldots, X_{n}\right\}$. Now we denote $T_{i j}^{s}$ by

$$
T_{i j}^{s}=g\left(T_{U i} U_{j}, X_{s}\right),
$$

where $1 \leq i, j \leq r$ and $1 \leq s \leq n$ (see [7]).

Similarly, we denote $A_{i j}^{\alpha}$ by

$$
A_{i j}^{\alpha}=g\left(A_{X i} X_{j}, U_{\alpha}\right) .
$$

where $1 \leq i, j \leq n$ and $1 \leq \alpha \leq r$. From [7], we use

$$
\delta(N)=\sum_{i=1}^{n} \sum_{k=1}^{r} g\left(\left(\nabla_{X_{i}} T\right)_{U_{k}} U_{k}, X_{i}\right) .
$$

Case I: Assume that $\xi$ is vertical.

Then from (3.1), we have

$$
2 \widehat{\tau}=\frac{c+3}{4} r(r-1)-\frac{c-1}{2}(r-1)-r^{2}\|H\|^{2}+\sum_{i, j=1}^{r} g\left(T_{U_{i}} U_{j}, T_{U_{i}} U_{j}\right) .
$$

Using (4.1) in the last equality and the symmetry of $T$, we can write

$$
2 \widehat{\tau}=\frac{c+3}{4} r(r-1)-\frac{c-1}{2}(r-1)-r^{2}\|H\|^{2}+\sum_{s=1}^{n} \sum_{i, j=1}^{r}\left(T_{i j}^{s}\right)^{2} .
$$


For a local orthonormal frame $\left\{X_{i}, U_{j}\right\}_{1 \leq i \leq n, 1 \leq j \leq r}$ on $M(c)$, such that the horizontal and vertical distributions are spanned by $\left\{X_{i},\right\}_{1 \leq i \leq n}$ and $\left\{U_{j}\right\}_{1 \leq j \leq r}$, respectively, we know from [7] that

$$
\begin{aligned}
& \sum_{s=1}^{n} \sum_{i, j=1}^{r}\left(T_{i j}^{s}\right)^{2}=\frac{1}{2} r^{2}\|H\|^{2}+\frac{1}{2} \sum_{s=1}^{n}\left[T_{11}^{s}-T_{22}^{s}-\ldots-T_{r r}^{s}\right]^{2} \\
& \quad+2 \sum_{s=1}^{n} \sum_{j=2}^{r}\left(T_{1 j}^{s}\right)^{2}-2 \sum_{s=1}^{n} \sum_{2 \leq i<j \leq r}^{r}\left[T_{i i}^{s} T_{j j}^{s}-\left(T_{i j}^{s}\right)^{2}\right] .
\end{aligned}
$$

So using the above equality in (4.4), we get

$$
\begin{gathered}
2 \widehat{\tau}=\frac{c+3}{4} r(r-1)-\frac{c-1}{2}(r-1) \\
-\frac{1}{2} r^{2}\|H\|^{2}+\frac{1}{2} \sum_{s=1}^{n}\left[T_{11}^{s}-T_{22}^{s}-\ldots-T_{r r}^{s}\right]^{2} \\
+2 \sum_{s=1}^{n} \sum_{j=2}^{r}\left(T_{1 j}^{s}\right)^{2}-2 \sum_{s=1}^{n} \sum_{2 \leq i<j \leq r}^{r}\left[T_{i i}^{s} T_{j j}^{s}-\left(T_{i j}^{s}\right)^{2}\right] .
\end{gathered}
$$

Then from the last equality, we have

$$
\begin{gathered}
2 \widehat{\tau} \geq \frac{c+3}{4} r(r-1)-\frac{c-1}{2}(r-1) \\
-\frac{1}{2} r^{2}\|H\|^{2}-2 \sum_{s=1}^{n} \sum_{2 \leq i<j \leq r}^{r}\left[T_{i i}^{s} T_{j j}^{s}-\left(T_{i j}^{s}\right)^{2}\right] .
\end{gathered}
$$

Furthermore, from (2.1), taking $U=W=U_{i}, V=F=U_{j}$ and using (4.1) we can write

$$
\begin{gathered}
2 \sum_{2 \leq i<j \leq r} R\left(U_{i}, U_{j}, U_{j}, U_{i}\right)=2 \sum_{2 \leq i<j \leq r} \widehat{R}\left(U_{i}, U_{j}, U_{j}, U_{i}\right) \\
\quad+2 \sum_{s=1}^{n} \sum_{2 \leq i<j \leq r}\left[T_{i i}^{s} T_{j j}^{s}-\left(T_{i j}^{s}\right)^{2}\right] .
\end{gathered}
$$

In view of the last equality, (4.6) can be written as

$$
\begin{gathered}
2 \widehat{\tau} \geq \frac{c+3}{4} r(r-1)-\frac{c-1}{2}(r-1)-\frac{1}{2} r^{2}\|H\|^{2} \\
+2 \sum_{2 \leq i<j \leq r} \widehat{R}\left(U_{i}, U_{j}, U_{j}, U_{i}\right)-2 \sum_{2 \leq i<j \leq r} R\left(U_{i}, U_{j}, U_{j}, U_{i}\right) .
\end{gathered}
$$


Then using the equality

$$
2 \widehat{\tau}=2 \sum_{2 \leq i<j \leq r} \widehat{R}\left(U_{i}, U_{j}, U_{j}, U_{i}\right)+2 \sum_{j=1}^{r} \widehat{R}\left(U_{1}, U_{j}, U_{j}, U_{1}\right),
$$

in view of (4.7), we have

$$
\begin{aligned}
& 2 \widehat{\operatorname{Ric}}\left(U_{1}\right) \geq \frac{c+3}{4} r(r-1)-\frac{c-1}{2}(r-1) \\
& -\frac{1}{2} r^{2}\|H\|^{2}-2 \sum_{2 \leq i<j \leq r} R\left(U_{i}, U_{j}, U_{j}, U_{i}\right) .
\end{aligned}
$$

Since $M$ is a Sasakian space form, its curvature tensor $R$ satisfies the equality (2.4). So we obtain

$$
\widehat{\operatorname{Ric}}\left(U_{1}\right) \geq \frac{c+3}{4}(r-1)+\frac{c-1}{4}\left\{(2-r) \eta\left(U_{1}\right)^{2}-1\right\}-\frac{1}{4} r^{2}\|H\|^{2} .
$$

Hence we state the following theorem:

Theorem 4.1. Let $\pi: M(c) \rightarrow B$ be an anti-invariant Riemannian submersion from a Sasakian space form $(M(c), g)$ onto a Riemannian manifold $\left(B, g^{\prime}\right)$ such that $\xi$ is vertical. Then

$$
\widehat{\operatorname{Ric}}\left(U_{1}\right) \geq \frac{c+3}{4}(r-1)-\frac{c-1}{4}\left\{(r-2) \eta\left(U_{1}\right)^{2}+1\right\}-\frac{1}{4} r^{2}\|H\|^{2} .
$$

The equality case of the inequality holds if and only if

$$
\begin{aligned}
& T_{11}^{s}=T_{22}^{s}+\ldots+T_{r r}^{s}, \\
& T_{1 j}=0, \quad j=2, \ldots, r .
\end{aligned}
$$

On the other hand, using (4.2) and Lemma 2.3, the equation (3.3) can be rewritten as

$$
2 \tau^{*}=\frac{c+3}{4} n(n-1)+\frac{3}{4}(c-1)(n+\operatorname{tr}(\phi B))-3 \sum_{\alpha=1}^{r} \sum_{i, j=1}^{n}\left(A_{i j}^{\alpha}\right)^{2} .
$$

Since $A$ is anti-symmetric on $\chi^{\mathcal{H}}(M(c))$, the above equality turns into

$$
\begin{aligned}
2 \tau^{*} & =\frac{c+3}{4} n(n-1)+\frac{3}{4}(c-1)(n+\operatorname{tr}(\phi B)) \\
& -6 \sum_{\alpha=1}^{r} \sum_{j=2}^{n}\left(A_{1 j}^{\alpha}\right)^{2}-6 \sum_{\alpha=1}^{r} \sum_{2 \leq i<j \leq n}\left(A_{i j}^{\alpha}\right)^{2} .
\end{aligned}
$$


Furthermore, from (2.2), taking $X=H=X_{i}, Y=Z=X_{j}$ and using (4.2), we have

$$
\begin{gathered}
2 \sum_{2 \leq i<j \leq n} R\left(X_{i}, X_{j}, X_{j}, X_{i}\right)=2 \sum_{2 \leq i<j \leq n} R^{*}\left(X_{i}, X_{j}, X_{j}, X_{i}\right) \\
+6 \sum_{\alpha=1}^{r} \sum_{2 \leq i<j \leq n}\left(A_{i j}^{\alpha}\right)^{2} .
\end{gathered}
$$

If we consider the last equality in (4.9), then we get

$$
\begin{aligned}
& 2 \tau^{*}=\frac{c+3}{4} n(n-1)+\frac{3}{4}(c-1)(n+\operatorname{tr}(\phi B))-6 \sum_{\alpha=1}^{r} \sum_{j=2}^{n}\left(A_{1 j}^{\alpha}\right)^{2} \\
& \quad+2 \sum_{2 \leq i<j \leq n} R^{*}\left(X_{i}, X_{j}, X_{j}, X_{i}\right)-2 \sum_{2 \leq i<j \leq n} R\left(X_{i}, X_{j}, X_{j}, X_{i}\right) .
\end{aligned}
$$

Since $M$ is a Sasakian space form, its curvature tensor $R$ satisfies the equality (2.4). Then we have

$$
\begin{aligned}
2 \operatorname{Ric}^{*}\left(X_{1}\right)= & \frac{c+3}{2}(n-1)+\frac{3}{4}(c-1)\left\|C X_{1}\right\|^{2} \\
& -6 \sum_{\alpha=1}^{r} \sum_{j=2}^{n}\left(A_{1 j}^{\alpha}\right)^{2} .
\end{aligned}
$$

So we can write

$$
\operatorname{Ric}^{*}\left(X_{1}\right) \leq \frac{c+3}{2}(n-1)+\frac{3}{4}(c-1)\left\|C X_{1}\right\|^{2} .
$$

Hence we obtain the following theorem:

Theorem 4.2. Let $\pi: M(c) \rightarrow B$ be an anti-invariant Riemannian submersion from a Sasakian space form $(M(c), g)$ onto a Riemannian manifold $\left(B, g^{\prime}\right)$ such that $\xi$ is vertical. Then

$$
\operatorname{Ric}^{*}\left(X_{1}\right) \leq \frac{c+3}{4}(n-1)+\frac{3}{4}(c-1)\left\|C X_{1}\right\|^{2} .
$$

The equality case of the inequality holds if and only if

$$
A_{1 j}=0, \quad j=2, \ldots, n .
$$

Since

$$
2 \tau=\sum_{s=1}^{n} \operatorname{Ric}\left(X_{s}, X_{s}\right)+\sum_{k=1}^{r} \operatorname{Ric}\left(U_{k}, X_{k}\right)
$$




$$
\begin{gathered}
2 \tau=\sum_{j, k=1}^{r} R\left(U_{j}, U_{k}, U_{k}, U_{j}\right)+\sum_{i=1}^{n} \sum_{k=1}^{r} R\left(X_{i}, U_{k}, U_{k}, X_{i}\right) \\
+\sum_{i, s=1}^{n} R\left(X_{i}, X_{s}, X_{s}, X_{i}\right)+\sum_{s=1}^{n} \sum_{j=1}^{r} R\left(U_{j}, X_{s}, X_{s}, U_{j}\right),
\end{gathered}
$$

where $\tau$ is the scalar curvature of $M(c)$. Since $M(c)$ is a Sasakian space form, using (4.11) and (2.4), we find

$$
2 \tau=\frac{c+3}{4}(r(r-1)+n(n-1)+2 n r)+\frac{c-1}{4}(4(r-1)+n+3 \operatorname{tr} \phi B) .
$$

On the other hand, from the Gauss-Codazzi type equations (2.1), (2.2) and (2.3), we have

$$
\begin{gathered}
2 \tau=2 \widehat{\tau}+2 \tau^{*}+r^{2}\|H\|^{2}+\sum_{k, j=1}^{r} g\left(T_{U_{k}} U_{j}, T_{U_{k}} U_{j}\right) \\
+3 \sum_{i, s=1}^{n} g\left(A_{X_{i}} X_{s}, A_{X_{i}} X_{s}\right)-\sum_{i=1}^{n} \sum_{k=1}^{r} g\left(\left(\nabla_{X_{i}} T\right)_{U_{k}} U_{k}, X_{i}\right) \\
+\sum_{i=1}^{n} \sum_{k=1}^{r}\left\{g\left(T_{U_{k}} X_{i}, T_{U_{k}} X_{i}\right)-g\left(A_{X_{i}} U_{k}, A_{X_{i}} U_{k}\right)\right\}-\sum_{s=1}^{n} \sum_{j=1}^{r} g\left(\left(\nabla_{X_{s}} T\right)_{U_{j}} U_{j}, X_{s}\right) \\
+\sum_{s=1}^{n} \sum_{j=1}^{r}\left\{g\left(T_{U_{j}} X_{s}, T_{U_{j}} X_{s}\right)-g\left(A_{X_{s}} U_{j}, A_{X_{s}} U_{j}\right)\right\} .
\end{gathered}
$$

Using (4.5) and (4.3), we get

$$
\begin{gathered}
2 \tau=2 \widehat{\tau}+2 \tau^{*}+\frac{1}{2} r^{2}\|H\|^{2}-\frac{1}{2} \sum_{s=1}^{n}\left[T_{11}^{s}-T_{22}^{s}-\ldots-T_{r r}^{s}\right]^{2} \\
-2 \sum_{s=1}^{n} \sum_{j=2}^{r}\left(T_{1 j}^{s}\right)^{2}+2 \sum_{s=1}^{n} \sum_{2 \leq j<k \leq r}^{r}\left[T_{j j}^{s} T_{k k}^{s}-\left(T_{j k}^{s}\right)^{2}\right]+6 \sum_{\alpha=1}^{r} \sum_{s=2}^{n}\left(A_{1 s}^{\alpha}\right)^{2} \\
+6 \sum_{\alpha=1}^{r} \sum_{2 \leq i<s \leq n}\left(A_{i s}^{\alpha}\right)^{2}+\sum_{i=1}^{n} \sum_{k=1}^{r}\left\{g\left(T_{U_{k}} X_{i}, T_{U_{k}} X_{i}\right)-g\left(A_{X_{i}} U_{k}, A_{X_{i}} U_{k}\right)\right\} \\
-2 \delta(N)+\sum_{s=1}^{n} \sum_{j=1}^{r}\left\{g\left(T_{U_{j}} X_{s}, T_{U_{j}} X_{s}\right)-g\left(A_{X_{s}} U_{j}, A_{X_{s}} U_{j}\right)\right\} .
\end{gathered}
$$

By making use of (4.8), (4.10) and (4.12) in the last equality, we obtain 


$$
\begin{gathered}
\frac{c+3}{2} n r+\frac{c-1}{2}(3(r-1)-n) \\
+2 \sum_{k=1}^{r} R\left(U_{1}, U_{k}, U_{k}, U_{1}\right)+2 \sum_{s=1}^{n} R\left(X_{1}, X_{s}, X_{s}, X_{1}\right) \\
=2 \widehat{\operatorname{Ric}}\left(U_{1}\right)+2 \operatorname{Ric}^{*}\left(X_{1}\right)+\frac{1}{2} r^{2}\|H\|^{2}-\frac{1}{2} \sum_{s=1}^{n}\left[T_{11}^{s}-T_{22}^{s}-\ldots-T_{r r}^{s}\right]^{2} \\
-2 \sum_{s=1}^{n} \sum_{j=2}^{r}\left(T_{1 j}^{s}\right)^{2}+6 \sum_{\alpha=1}^{r} \sum_{s=2}^{n}\left(A_{1 s}^{\alpha}\right)^{2}+\sum_{i=1}^{n} \sum_{k=1}^{r}\left\{g\left(T_{U_{k}} X_{i}, T_{U_{k}} X_{i}\right)-g\left(A_{X_{i}} U_{k}, A_{X_{i}} U_{k}\right)\right\} \\
-2 \delta(N)+\sum_{s=1}^{n} \sum_{j=1}^{r}\left\{g\left(T_{U_{j}} X_{s}, T_{U_{j}} X_{s}\right)-g\left(A_{X_{s}} U_{j}, A_{X_{s}} U_{j}\right)\right\} .
\end{gathered}
$$

We denote

$$
\left\|T^{V}\right\|^{2}=\sum_{i=1}^{n} \sum_{k=1}^{r} g\left(T_{U_{k}} X_{i}, T_{U_{k}} X_{i}\right)
$$

and

$$
\left\|A^{H}\right\|^{2}=\sum_{i=1}^{n} \sum_{k=1}^{r} g\left(A_{X_{i}} U_{k}, A_{X_{i}} U_{k}\right)
$$

(see [7]).

Since $(M(c), g)$ is a Sasakian space form, from (2.4), we obtain the following theorem:

Theorem 4.3. Let $\pi: M(c) \rightarrow B$ be an anti-invariant Riemannian submersion from a Sasakian space form $(M(c), g)$ onto a Riemannian manifold $\left(B, g^{\prime}\right)$ such that $\xi$ is vertical. Then

$$
\begin{gathered}
\frac{c+3}{4}\{n r+n+r-2\}+\frac{c-1}{4}\{3 r-4-n \\
\left.-(r-2) \eta\left(U_{1}\right)^{2}+3\left\|C X_{1}\right\|^{2}\right\} \leq \widehat{\operatorname{Ric}}\left(U_{1}\right)+\operatorname{Ric}^{*}\left(X_{1}\right)+\frac{1}{4} r^{2}\|H\|^{2} \\
+3 \sum_{\alpha=1}^{r} \sum_{s=2}^{n}\left(A_{1 s}^{\alpha}\right)^{2}-\delta(N)+\left\|T^{V}\right\|^{2}-\left\|A^{H}\right\|^{2} .
\end{gathered}
$$

The equality case of the inequality holds if and only if

$$
\begin{aligned}
& T_{11}^{s}=T_{22}^{s}+\ldots+T_{r r}^{s}, \\
& T_{1 j}=0, \quad j=2, \ldots, r .
\end{aligned}
$$


Case II: Assume that $\xi$ is horizontal.

From (3.1), similar to Theorem 4.1, we can state the following theorem:

Theorem 4.4. Let $\pi: M(c) \rightarrow B$ be an anti-invariant Riemannian submersion from a Sasakian space form $(M(c), g)$ onto a Riemannian manifold $\left(B, g^{\prime}\right)$ such that $\xi$ is horizontal. Then

$$
\widehat{\operatorname{Ric}}\left(U_{1}\right) \geq \frac{c+3}{4}(r-1)-\frac{1}{4} r^{2}\|H\|^{2} .
$$

The equality case of the inequality holds if and only if

$$
\begin{aligned}
& T_{11}^{s}=T_{22}^{s}+\ldots+T_{r r}^{s}, \\
& T_{1 j}=0, \quad j=2, \ldots, r .
\end{aligned}
$$

From (3.2), similar to Theorem 4.2, we have the following theorem:

Theorem 4.5. Let $\pi: M(c) \rightarrow B$ be an anti-invariant Riemannian submersion from a Sasakian space form $(M(c), g)$ onto a Riemannian manifold $\left(B, g^{\prime}\right)$ such that $\xi$ is horizontal. Then

$$
\operatorname{Ric}^{*}\left(X_{1}\right) \leq \frac{c+3}{4}(n-1)+\frac{c-1}{4}\left\{(2-n) \eta\left(X_{1}\right)^{2}-1+3\left\|C X_{1}\right\|^{2}\right\} .
$$

The equality case of the inequality holds if and only if

$$
A_{1 j}=0, j=2, \ldots, n \text {. }
$$

Since $\xi$ is horizontal, from (4.11), we find

$$
2 \tau=\frac{c+3}{4}[r(r-1)+n(n-1)+2 n r]+\frac{c-1}{4}[n+3 \operatorname{tr} \phi B+4 r-7] .
$$

Using the above equation, (4.13), (4.5), (4.8), (4.10) and (4.3), we get

$$
\begin{gathered}
\frac{c+3}{2} n r+\frac{c-1}{2}(2 r-3) \\
+2 \sum_{k=1}^{r} R\left(U_{1}, U_{k}, U_{k}, U_{1}\right)+2 \sum_{s=1}^{n} R\left(X_{1}, X_{s}, X_{s}, X_{1}\right) \\
=2 \widehat{\operatorname{Ric}}\left(U_{1}\right)+2 \operatorname{Ric}^{*}\left(X_{1}\right)+\frac{1}{2} r^{2}\|H\|^{2}-\frac{1}{2} \sum_{s=1}^{n}\left[T_{11}^{s}-T_{22}^{s}-\ldots-T_{r r}^{s}\right]^{2} \\
-2 \sum_{s=1}^{n} \sum_{j=2}^{r}\left(T_{1 j}^{s}\right)^{2}+6 \sum_{\alpha=1}^{r} \sum_{s=2}^{n}\left(A_{1 s}^{\alpha}\right)^{2}-2 \delta(N)
\end{gathered}
$$




$$
\begin{aligned}
& +\sum_{s=1}^{n} \sum_{j=1}^{r}\left\{g\left(T_{U_{j}} X_{s}, T_{U_{j}} X_{s}\right)-g\left(A_{X_{s}} U_{j}, A_{X_{s}} U_{j}\right)\right\} \\
& +\sum_{i=1}^{n} \sum_{k=1}^{r}\left\{g\left(T_{U_{k}} X_{i}, T_{U_{k}} X_{i}\right)-g\left(A_{X_{i}} U_{k}, A_{X_{i}} U_{k}\right)\right\} .
\end{aligned}
$$

Hence in view of (2.4), we obtain the following theorem:

Theorem 4.6. Let $\pi: M(c) \rightarrow B$ be an anti-invariant Riemannian submersion from a Sasakian space form $(M(c), g)$ onto a Riemannian manifold $\left(B, g^{\prime}\right)$ such that $\xi$ is horizontal. Then

$$
\begin{gathered}
\frac{c+3}{4}\{n r+n+r-2\}+\frac{c-1}{4}\left\{2 r-4-(n-2) \eta\left(X_{1}\right)^{2}\right. \\
\left.+3\left\|C X_{1}\right\|^{2}\right\} \leq \widehat{\operatorname{Ric}}\left(U_{1}\right)+\operatorname{Ric}^{*}\left(X_{1}\right)+\frac{1}{4} r^{2}\|H\|^{2} \\
+3 \sum_{\alpha=1}^{r} \sum_{s=2}^{n}\left(A_{1 s}^{\alpha}\right)^{2}-\delta(N)+\left\|T^{V}\right\|^{2}-\left\|A^{H}\right\|^{2} .
\end{gathered}
$$

The equality case of the inequality holds if and only if

$$
\begin{aligned}
& T_{11}^{s}=T_{22}^{s}+\ldots+T_{r r}^{s}, \\
& T_{1 j}=0, \quad j=2, \ldots, r .
\end{aligned}
$$

\section{REFERENCES}

[1] P. Alegre, B.-Y. Chen, M. I. Munteanu, Riemannian submersions, $\delta$-invariants, and optimal inequality, Ann. Global Anal. Geom. 42 (2012), no. 3, 317-331.

[2] H. Aytimur and C. Özgür, Inequalities for submanifolds in statistical manifolds of quasiconstant curvature, Ann. Pol. Math., 121 (2018), no. 3, 197-215.

[3] D. E. Blair, Riemannian geometry of contact and symplectic manifolds, Second edition. Progress in Mathematics, 203. Birkhäuser Boston, Inc., Boston, MA, 2010.

[4] B.-Y. Chen, Riemannian submersions, minimal immersions and cohomology class, Proc. Japan Acad. Ser. A Math. Sci. 81 (2005), no. 10, 162-167 (2006).

[5] B.-Y. Chen, Examples and classification of Riemannian submersions satisfying a basic equality, Bull. Austral. Math. Soc. 72 (2005), no. 3, 391-402.

[6] M. Falciteli, S. Ianus A. M. Pastore, Riemannian submersions and Related Topics, World Scientific Publishing Co. Pte. Ltd (2004).

[7] M. Gülbahar, Ş. Eken Meriç and E. Kiliç, Sharp inequalities involving the Ricci curvature for Riemannian submersions, Kragujevac J. Math. 41 (2017), no. 2, 279-293.

[8] B. O'Neill, The fundamental equations of a submersion, Michigan Math. J. 13 (1966), 459-469. 
[9] B. O'Neill, Semi-Riemannian Geometry with Application to Relativity, Academic Press, New York, 1983.

[10] M. E. Aydın, A. Mihai, I. Mihai, Some Inequalities on submanifolds in statistical manifolds of constant curvature, Filomat 29 (2015), no. 3, 465-477.

[11] B.-Y. Chen, Some pinching and classification theorems for minimal submanifolds, Arch. Math. (Basel) 60 (1993), 568-578.

[12] B.-Y. Chen, Relations between Ricci curvature and shape operator for submanifolds with arbitrary codimensions, Glasg. Math. J. 41 (1999), no. 1, 33-41.

[13] B.-Y. Chen, Pseudo-Riemannian Geometry, $\delta$-invariants and Applications, World Scientific Publishing Co. Pte. Ltd., Hackensack, NJ, 2011.

[14] İ. Küpeli Erken and C. Murathan, Anti-invariant Riemannian submersions from Sasakian manifolds, arXiv:1302.4906, 2013

[15] A. Mihai, Modern Topics in Submanifold Theory, Editura Universitatii Bucuresti, Bucharest, 2006.

[16] A. Mihai, I. Mihai, Curvature invariants for statistical submanifolds of Hessian manifolds of constant Hessian curvature, Mathematics, (2018), 6, 44.

[17] A. Mihai and C. Özgür, Chen inequalities for submanifolds of real space forms with a semi-symmetric metric connection, Taiwanese J. Math. 14 (2010), no. 4, 1465-1477.

[18] A. Mihai and C. Özgür, Chen inequalities for submanifolds of complex space forms and Sasakian space forms endowed with semi-symmetric metric connections, Rocky Mountain J. Math. 41 (2011), no. 5, 1653-1673.

[19] C. Özgür, B. Y. Chen inequalities for submanifolds of a Riemanian manifold of quasiconstant curvature, Turk. J. Math. 35 (2011) 501-509.

[20] B. Şahin, Anti-invariant Riemannian submersions from almost Hermitian manifolds, Cent. Eur. J. Math. 8 (2010), no. 3, 437-447.

[21] B. Şahin, Chen's first inequality for Riemannian maps, Ann. Polon. Math. 117 (2016), no. 3, 249-258.

Balikesir University, Department of Mathematics, 10145 Balikesir, TURKEY

E-mail address: hulya.aytimur@balikesir.edu.tr

Balikesir University, Department of Mathematics, 10145 Balikesir, TURKEY

E-mail address: cozgur@balikesir.edu.tr 